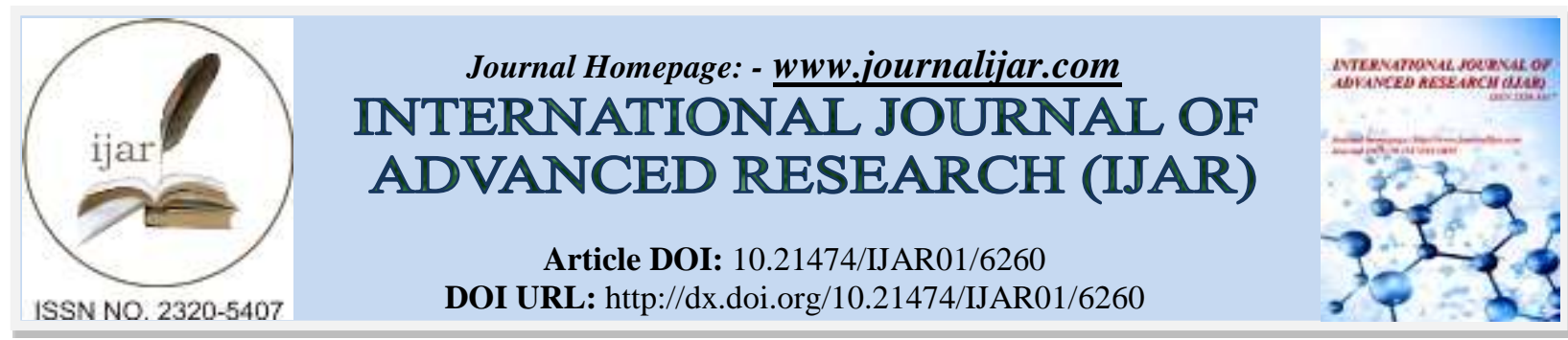

RESEARCH ARTICLE

\title{
DOES TIMING OF DIGESTIVE ENDOSCOPY AFFECT MORBIDITY AND MORTALITY OF UPPER GASTROINTESTINAL BLEEDING IN A MALAGASY POPULATION?
}

\author{
"Rakotondrainibe Aurélia, Rakotomena S. Davidà, Randriamizao H.M. Richard, Avotsihoarana T. \\ Hélianthe, Rajaonera A. Tovohery and Rakotoarison R. C. Nicole. \\ Faculté de Médecine d'Antananarivo - Université d'Antananarivo, Madagascar.
}

\section{Manuscript Info}

Manuscript History

Received: 09 November 2017

Final Accepted: 11 December 2017

Published: January 2018

Key words:-

Gastrointestinal endoscopy,

Gastrointestinal hemorrhage, Morbidity, Mortality.

\section{Abstract}

Background: Gastrointestinal bleeding remains an emergency, where digestive endoscopy is unavoidable. Digestive endoscopy should be performed as soon as possible, because it can affect the morbidity and mortality of patients. The aims of this study were to assess the timing of the digestive endoscopy and its correlations with morbidity and mortality in upper gastrointestinal bleeding in a hospital in Antananarivo (Madagascar).

Methods: In a five-year retrospective study (from 2010 to 2014), records of patients with upper gastrointestinal bleeding, investigated by digestive endoscopy were included. The timing of this endoscopy has been studied; as well as its correlations (Spearman's test) with the morbidity and mortality of digestive hemorrhages.

Results: During the study, 711 cases were selected. The patients were mostly men with an average age of $48.6 \pm 14.4$ years old. Non variceal upper gastrointestinal bleeding represented $87.8 \%$ of the patients. The timing of upper gastrointestinal fibroscopy was $3.5 \pm 1.6$ days, of which $4.6 \%$ was performed within 24 hours and $20.9 \%$ within 48 hours after admission. The lenght of stay was correlated with a delay of realization within 48 hours; the mortality decreased when this endoscopy was carried out within 24 hours.

Conclusion: To follow the current recommendations on the timing of the digestive endoscopy in digestive upper gastrointestinal bleeding is essential. The goal of early delay is still difficult for Malagasy patients. Nevertheless, its realization in the $24 \mathrm{~h}-48 \mathrm{~h}$ was significant on the duration of stay and the mortality.

Copy Right, IJAR, 2018,. All rights reserved.

\section{Introduction:-}

Les hémorragies digestives hautes (HDH) sont grevées d'une mortalité variant de 2,5 à $10 \%$ pour les hémorragies non variqueuses (HDHNV) et de 5 à $30 \%$ pour les hémorragies d'origine variqueuse (HDHV). ${ }^{\mathbf{2}, 4}$ Le taux de resaignement est de l'ordre de $10,4 \% .{ }^{2}$ Les principes de prise en charge des hémorragies digestives reposent entre autres sur la compensation de la perte volémique, la transfusion sanguine, sous un monitorage hémodynamique rapproché. ${ }^{7}$ De plus, une hémorragie digestive représente une urgence diagnostique et thérapeutique où l'endoscopie digestive tient une place prépondérante. ${ }^{14}$ L'endoscopie digestive haute ou fibroscopie œso-gastro-duodénale (FOGD) doit être réalisée dans les premières 12 à 24 heures pour les HDHV et les HDHNV respectivement, selon 
les recommandations internationales actuelles. ${ }^{7,17}$ Cet examen étant incontournable, sa réalisation dans l'urgence permet une détection rapide des lésions à haut risque de saignement et un traitement endoscopique précoce. ${ }^{7,18} \mathrm{Le}$ délai optimal de la réalisation de la FOGD reste controversé voire empirique dans les HDHNV et les HDHV. ${ }^{\mathbf{1 , 4}} \mathrm{La}$ réalisation de cet examen peut se faire dès l'admission du patient, mais peut varier selon le jour d'entrée ; le triage permettra de déterminer ceux qui ont besoin de cet examen dans l'immédiat. ${ }^{11,12}$ Le délai d'application de la FOGD peut ne pas avoir d'impact sur la morbi-mortalité des patients et peut entraîner une diminution de la durée d'hospitalisation et la nécessité d'une transfusion sanguine. ${ }^{7,16}$

Dans les centres hospitaliers à Madagascar, les soins et les dépenses de santé sont à la charge des patients. Il en est de même pour la prise en charge des examens complémentaires, tout comme l'endoscopie digestive. Le fait que les patients subviennent eux-mêmes à leurs soins hospitaliers, du point de vue pécuniaire, la réalisation des divers examens complémentaires est variable selon le statut financier du patient et certains examens peuvent être retardés. D'où les objectifs de cette étude : de déterminer le délai de réalisation de la FOGD et d'en ressortir les corrélations avec la morbi-mortalité, chez des patients ayant présenté une hémorragie digestive haute, dans un centre hospitalier d'Antananarivo-Madagascar.

\section{Methodologie:-}

Une étude rétrospective a été réalisée au Centre Hospitalier Universitaire Joseph Ravoahangy Andrianavalona (CHU JRA) à Antananarivo - Madagascar, sur une période de cinq ans (de 2010 à 2014). Les dossiers des patients admis au service de réanimation chirurgicale après un passage à l'accueil des urgences, pour hémorragies digestives hautes (variqueuses ou non) ont été étudiés. Les dossiers des patients ayant présenté une hémorragie digestive non explorée par une endoscopie digestive n'ont pas été inclus ; ceux dont les données portant sur la FOGD étaient présentes dans le dossier l'ont été. Les patients sortis contre avis médical du service ont été exclus.

Le critère de jugement principal a été l'impact du délai de réalisation de la FOGD avec la morbi-mortalité. Les données analysées étaient les critères démographiques, les caractéristiques de l'hémorragie digestive, les paramètres hémodynamiques à l'admission, le délai de réalisation de la FOGD, la morbidité (récidive de l'hémorragie lors de l'hospitalisation, nécessité de chirurgie d'hémostase) et la mortalité en réanimation chirurgicale. Les corrélations étudiées étaient celles entre le délai de réalisation de la FOGD et la morbi-mortalité.

Les variables quantitatives ont été exprimées en moyenne avec écartype, les variables qualitatives en pourcentage. Le test de corrélation de Pearson (SigmaStat ${ }^{\circledR}$ 3.5) a été utilisé pour déterminer les diverses corrélations. Une valeur de $\mathrm{p}$ inférieure à 0,05 a été considérée comme significative.

\section{Resultats:-}

Durant la période d'étude, 1030 dossiers ont été analysés. Dans 311 dossiers, aucune donnée de FOGD ne figurait ; aussi, 719 dossiers de patients ont été considérés. Huit patients étaient sortis contre avis médical, 711 dossiers ont été ainsi retenus. Les principales caractéristiques de la population sont représentées dans le tableau I.

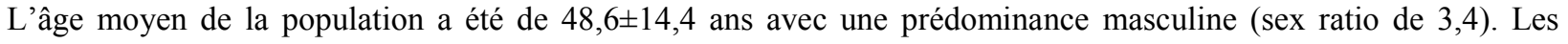
patients ASA 1 étaient les plus nombreux $(74,1 \%)$.

Quatre-vingt-sept patients $(12,2 \%)$ ont présenté une hémorragie digestive haute variqueuse contre $87,8 \%$ d'hémorragie digestive non variqueuse. La présentation la plus fréquente de l'hémorragie a été le méléna (42,6\%). L'accident hémorragique a été inaugural dans $73,1 \%$ des cas. Le nombre d'accidents hémorragiques a été de 1,3 $\pm 0,6$ (Tableau 1).

Portant sur la fibroscopie digestive, le délai de réalisation était de $3,5 \pm 1,6$ jours avec un maximum de 15 jours. Pour 33 patients $(4,6 \%)$ cet examen a été réalisé dans les 24 heures suivant l'admission et pour 149 patients $(20,9 \%)$ dans les 48 heures.

L'état hémodynamique à l'admission était caractérisé par une pression artérielle systolique (PAS) à 113,6 $\pm 23,8$ $\mathrm{mmHg}$, une pression artérielle moyenne $(\mathrm{PAM})$ à $81,3 \pm 17,5 \mathrm{mmHg}$ ainsi qu'une fréquence cardiaque (FC) à $91,4 \pm 15,0 \mathrm{bpm}$ et un indice de choc (IDC) à $0,8 \pm 0,3$. Le délai de réalisation de la FOGD n'a pas été influencé par les paramètres clinico-hémodynamiques sus-cités du patient à l'admission dans le service $(\mathrm{p}>0,05)$. 
La transfusion a été administrée dans $56,0 \%$ des cas avec une quantité moyenne de 1,8 $\pm 2,3$ poches transfusées (extrêmes de 1 à 15 poches, durant la durée de séjour). Lorsque la FOGD était précocement réalisée, la transfusion était quelque peu plus importante, sans être significative ( $\mathrm{p}=0,280$, Fig. 1). Il en a été de même lorsque cet examen endoscopique a été réalisé dans les 24 heures $(\mathrm{p}=0,207)$ ou dans les 48 heures $(\mathrm{p}=0,0608)$.

Dix-sept patients ont été opérés ou ont présenté une récidive hémorragique en intra-hospitalier. Le délai de réalisation de la FOGD n'avait pas de corrélation significative avec cette récidive hémorragique et la nécessité de chirurgie ( $\mathrm{p}=0,391$ ), même lorsqu'elle était réalisée dans les 24 heures ou les 48 heures (Fig. 2).

La durée de séjour en réanimation a été de $5,4 \pm 4,9$ jours. Bien qu'une réalisation précoce de la FOGD entrainait une durée d'hospitalisation moins longue, la corrélation n'était pas significative ( $\mathrm{p}=0,087$, Fig. 3), même lorsque la FOGD a été réalisée dans les 24 heures $(\mathrm{p}=0,401)$. La relation devient significative quand cet examen se faisait dans les 48 heures $(\mathrm{p}=0,00214)$.

Dix-huit décès ont été déplorés. Le délai de réalisation de cet examen, en général, n'avait pas d'influences sur la mortalité $(\mathrm{p}=0,308)$. Néanmoins, celle-ci était moindre lorsque la FOGD a été réalisée dans les 24 heures $(\mathrm{p}=0,014)$ (Fig. 2).

\section{Discussion:-}

Des questions se posent dans les hémorragies digestives, notamment dans les HDHNV : si une endoscopie digestive réalisée précocement permet de décharger les patients à faible risque, de diminuer l'utilisation des ressources thérapeutiques et d'améliorer l'issue chez les patients à haut risque. ${ }^{15}$ Dans les HDHV, le délai de réalisation de cet examen a une corrélation variable avec la mortalité et varie selon les études. ${ }^{1,4}$ La question est alors si la FOGD précoce est bénéfique ? ${ }^{15}$

Dans l'étude que nous avons réalisée, le délai de réalisation de la FOGD a été variable selon les patients. Une faible proportion a pu la réaliser précocement (dans les 24 heures après l'admission). Par rapport aux corrélations avec la morbi-mortalité, ce délai, en général n'avait pas de relation significative avec la morbidité. Lorsqu'elle a été réalisée dans les 24 heures, elle avait un impact sur la mortalité et sur la durée de séjour si réalisée dans les 48 heures.

Il est établi de façon universelle que la FOGD doit se faire dans les 24 heures, «tout comme le délai thérapeutique dans l'infarctus du myocarde qui doit se faire dans l'heure ${ }^{15}$ Le moment optimal pour la réalisation de FOGD dans les hémorragies variqueuses est encore un sujet à controverse. ${ }^{5}$ Réaliser trop tôt une FOGD ne permettrait pas une réanimation adéquate et pourrait résulter en de pires conséquences du point de vue morbi-mortalité. De plus si réalisée trop tôt, les ressources matérielles pourraient être incomplètes pour l'endoscopiste. Cependant si retardée, le pronostic pourrait être aggravé à cause d'un saignement persistant. ${ }^{8}$

Un délai de 24 heures a été avancé pour diminuer la durée de séjour hospitalier et probablement le recours à la chirurgie tout en réduisant le taux de mortalité et de resaignement chez les patients à haut risque. ${ }^{8}$ Cette endoscopie sera bénéfique après stabilisation hémodynamique et optimisation des voies aériennes supérieures. ${ }^{18}$

Le délai de réalisation de l'endoscopie est un facteur déterminant de la mortalité, du resaignement, de la nécessité d'une intervention (chirurgicale ou radiologique interventionnelle). ${ }^{12,17}$ La réalisation de la fibroscopie à moins de 6 heures jusqu'à la $24^{\text {ème }}$ heure était corrélée avec la mortalité. ${ }^{13}$ Le moment de la réalisation de l'endoscopie digestive est un facteur prédictif de l'issue du patient (odds ratio ajusté de 0,55 per 6 heures), lorsqu'elle est réalisée dans les six heures suivant l'admission du patient présentant une hémorragie digestive haute non variqueuse. ${ }^{7}$ La réalisation de la FOGD à plus de 24 heures est un facteur de mortalité, augmentant de 1,18 à 1,38 le risque de décès dans les $\mathrm{HDH}$ (d'origine variqueuse ou non). ${ }^{18}$ Plus ce délai augmente, plus le pronostic du patient s'assombrit.

Dans le cadre des HDHNV, la mortalité à 28 jours décroit selon que la FOGD ait été réalisée urgemment. ${ }^{2}$ Le délai de la réalisation de la FOGD dans les HDHNV a un impact sur la mortalité intra-hospitalière et à 30 jours selon la classe ASA du patient et la stabilité hémodynamique. ${ }^{9}$ Afin de ne pas influer sur la mortalité dans les HDHNV, Laursen et al. ${ }^{9}$ préconisent la réalisation de la FOGD dans les 12 à 24 premières heures pour les patients ASA 1 et 2 ; dans les 12 à 36 heures après l'admission à l'hôpital pour les patients ASA 3 à 5 et dans les $6-12$ heures à 24 heures pour les patients présentant une instabilité hémodynamique. Au-delà de ces fourchettes, la mortalité est plus importante. ${ }^{9}$ 
Dans les HDHV, Hsu et al. ${ }^{4}$ retrouvent que le délai de réalisation de la FOGD était significativement plus long chez les patients décédés (17,7 heures versus 12,9 heures, avec une médiane de 13,4 heures). Le retard de cet examen augmente de 3,67 le risque de décès mais d'autres facteurs y sont également corrélés tels que les scores spécifiques dans les HDHV, la présence d'hépatocarcinome ou l'hématémèse à la présentation de l'hémorragie digestive. ${ }^{\mathbf{1} 4}$ Pour les patients à faible risque, le délai de réalisation de la FOGD n'était pas déterminant de la mortalité $(16,7 \pm 18,5$ heures pour les patients décédés), contrairement aux patients à haut risque $(21,8 \pm 10,1$ heures) par rapport aux survivants (11,0 $\pm 10,6$ heures et 17,6 $\pm 15,7$ heures) ${ }^{11}$ Le moment de réalisation de l'endoscopie digestive n'est pas uniquement corrélé avec la mortalité, mais également avec d'autres paramètres de morbidité comme le resaignement ou la chirurgie, et cela varie également selon les études. Lorsque la FOGD est réalisée dans les 12 heures après l'admission du patient, la morbidité des HDH s'accroit de 5 fois par rapport aux patients pour lesquels le délai de réalisation de la FOGD dépassait les 12 heures. $^{7}$

Cette FOGD réalisée dans l'urgence est non corrélée avec le resaignement, mais plutôt avec une transfusion plus importante et le recours à la chirurgie. ${ }^{2}$ Dans le cadre du caractère urgent de l'endoscopie, l'hémodynamique peut être instable avec présentation d'un état de choc, ce qui pourrait augmenter cette morbidité. ${ }^{2}$ Dans les HDHV, le risque de resaignement à six semaines était lié, en plus de la présence de thrombose de la veine porte, au délai de réalisation de la FOGD (risque multiplié par 1,015). ${ }^{4}$

A contrario, le délai de réalisation de la FOGD peut ne pas être un facteur de mortalité dans les HDHV ${ }^{\mathbf{1}}$. Bien qu'une endoscopie, notamment à visée thérapeutique soit nécessaire, le délai de réalisation de celle-ci n'influe pas sur la prévention de resaignement et la survie. ${ }^{5}$

Une endoscopie réalisée dans les six heures diminue de manière non significative les complications d'ordre gastrointestinal par rapport à la réalisation effectuée dans les 24 heures ${ }^{16}$. Dans les pathologies ulcéreuses ou variqueuses à haut risque de saignement, la précocité de la réalisation de la FOGD est liée à la baisse du resaignement, ce dernier était plutôt lié à la sévérité de la pathologie, à la source de l'hémorragie, à la présence de stigmate. ${ }^{3}$

Le concept de «le plus tôt serait le mieux » n'est pas vraiment une réalité pour les HDHV car la mortalité ne décroît pas lorsque la FOGD est réalisée plus tôt ; Hsu et al. ${ }^{4}$ exposent un délai optimal de 15 heures. Dans les HDHNV, il est recommandé que la FOGD se fasse après des mesures de réanimation. ${ }^{6}$ Le délai de réalisation varie selon des facteurs cliniques, cependant la FOGD doit se faire dans les 24 heures devant une cirrhose, des signes de malignité, une hématémèse, une hypotension artérielle, une tachycardie, un état de choc. ${ }^{6}$

Portant sur la durée d'hospitalisation, une endoscopie réalisée de manière précoce est associée à un séjour hospitalier plus court et moins coûteux. ${ }^{17}$ Une réalisation précoce de la FOGD permet de diminuer le coût de l'hospitalisation et permet de décharger de $46 \%$ les patients qui n'ont pas de signes de gravité dans les HDHNV. ${ }^{\mathbf{1 0}}$

Ainsi, le délai de réalisation est variable selon les études, avec un impact différent sur la morbi-mortalité des hémorragies digestives selon les études. Dans notre milieu, les patients sont de prime abord accueillis au service des urgences puis dirigés vers les secteurs ou la réanimation chirurgicale, selon le risque. Aussi, les dépenses engendrées par les soins de cette pathologie se présentent dès l'accueil aux urgences, où la priorité est la prise en charge hémodynamique. Dans notre étude, une faible proportion des patients a pu réaliser la FOGD dans les 24 heures et dans les 48 heures après leur admission. Néanmoins, ces délais de 24 heures et 48 heures permettraient de diminuer la durée de séjour et la mortalité intra-hospitalière. Des résultats ont été ressortis, mais cette étude est limitée par son caractère monocentrique. De plus d'autres facteurs retardant la réalisation de la FOGD auraient pu être analysés.

\section{Conclusion:-}

La fibroscopie digestive dans la prise en charge des hémorragies digestives hautes tient une place importante. En plus de sa propriété diagnostique et thérapeutique, le délai de sa réalisation peut avoir une corrélation avec la durée de séjour et la mortalité. Dans un contexte où les patients doivent payer de leurs propres ressources leurs soins hospitaliers, le facteur financier (bien que non analysé) aurait pu être un facteur de retard de réalisation de la FOGD. Afin de suivre les recommandations actuelles pour la réaliser au plus tôt, des mesures devraient être mises en route pour faciliter l'accès à cet examen. Néanmoins, la morbi-mortalité n'a pas été impactée par le délai de réalisation de l'endoscopie digestive; la prise en charge des hémorragies digestives reste surtout focalisée sur la thérapeutique hémodynamique. 
Tableau:- Caractéristiques démographiques

\begin{tabular}{|l|l|l|l|}
\hline & & $\mathbf{n}$ & $\%$ \\
\hline Genre & Féminin & 163 & 22,9 \\
\hline Classe ASA & Masculin & 558 & 77,1 \\
\hline & ASA 1 & 527 & 74,1 \\
\hline & ASA 2 & 169 & 23,8 \\
\hline Hémorragie digestive haute & ASA 3 & 15 & 02,1 \\
\hline & Variqueuse & 86 & 12,2 \\
\hline Présentation de l'hémorragie & Non variqueuse & 87,8 \\
\hline & Hématémèse & 625 & 28,7 \\
\hline & Méléna & 204 & 42,6 \\
\hline Accident hémorragique & Hématémèse et méléna & 303 & 27,8 \\
\hline & Inaugural & 520 & 73,1 \\
\hline & Deux accidents & 21,3 \\
\hline
\end{tabular}

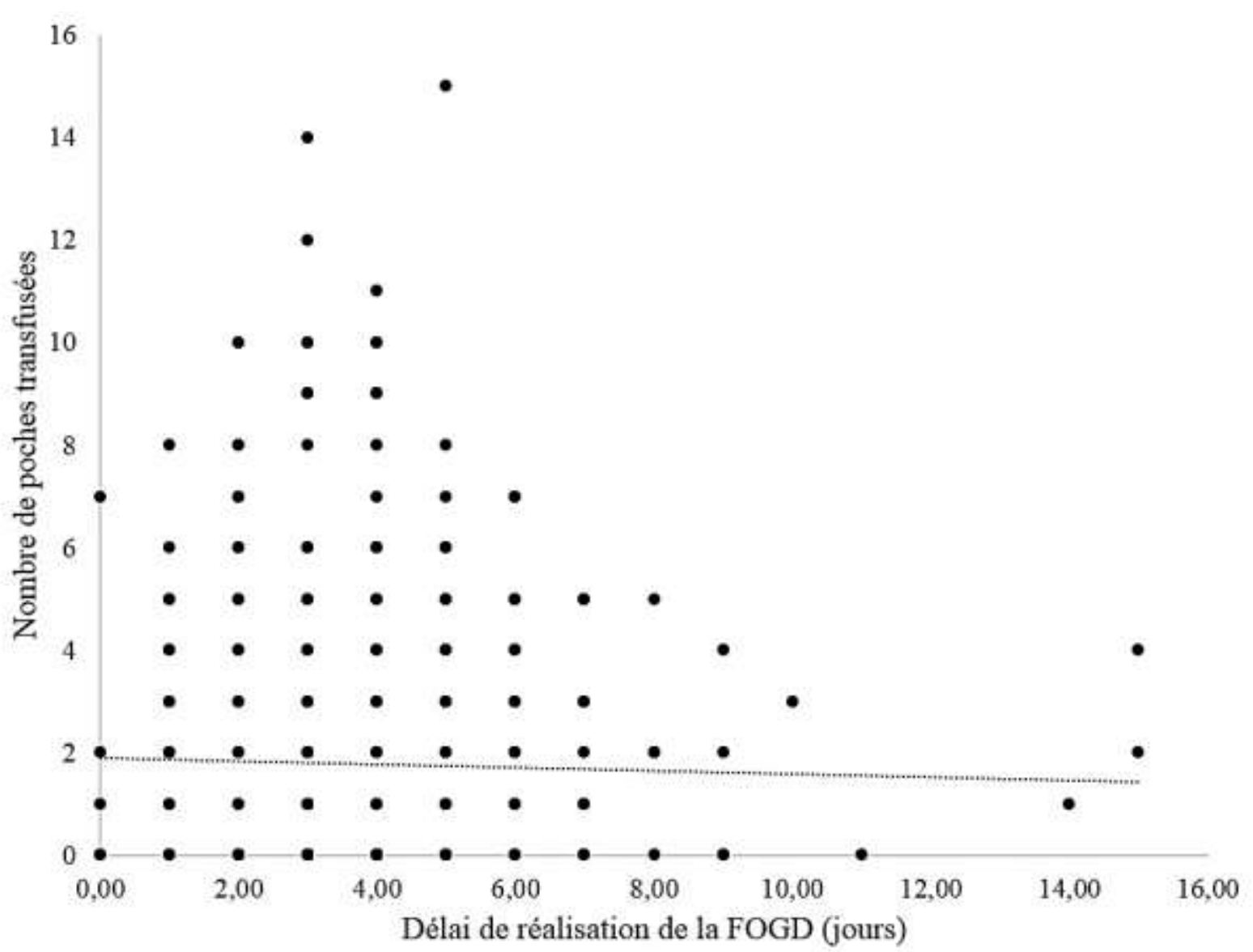

Figure 1:- Délai de réalisation de la FOGD et transfusion 


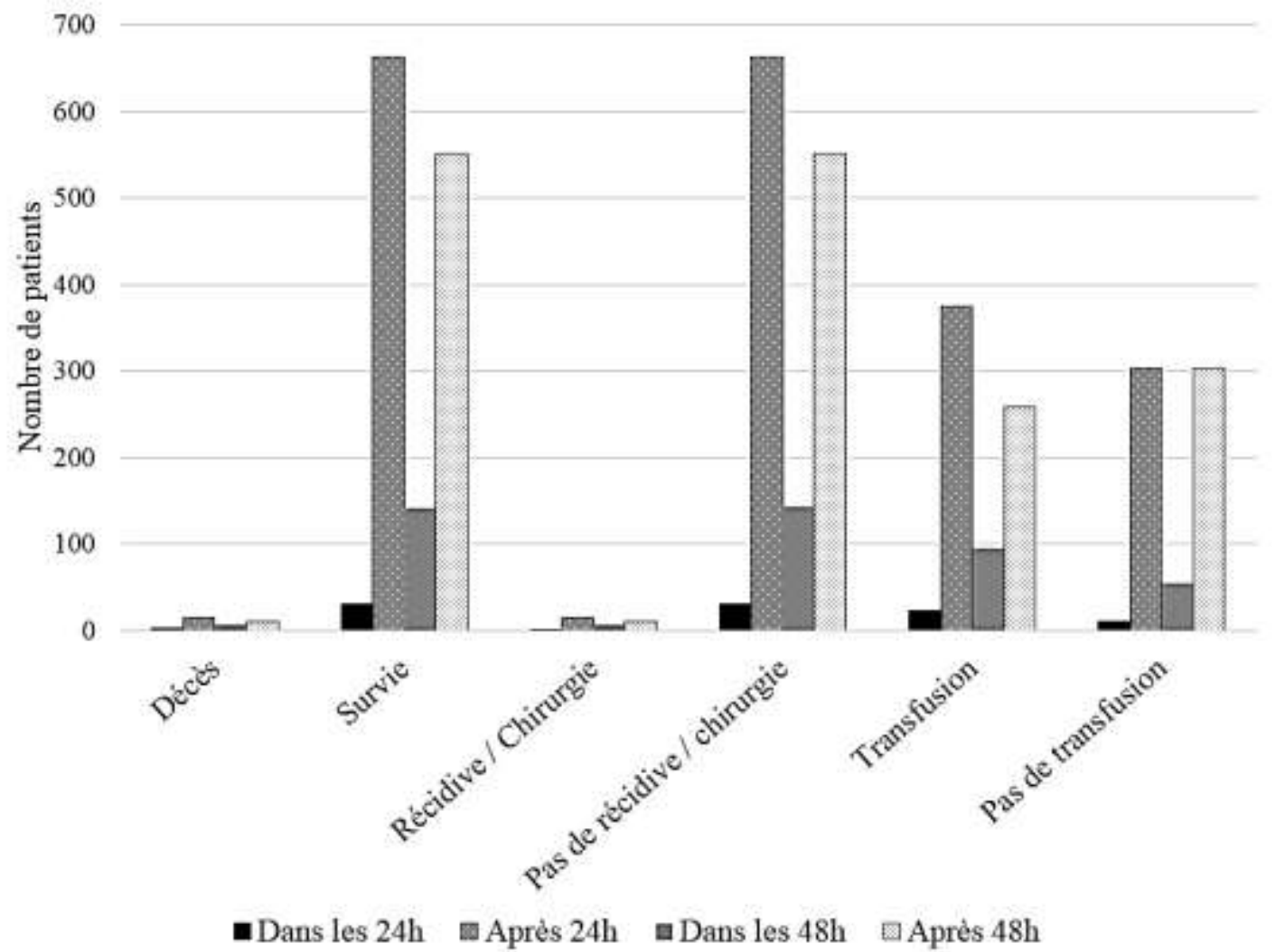

Figure 2:- Délai de réalisation de la FOGD et morbi-mortalité.

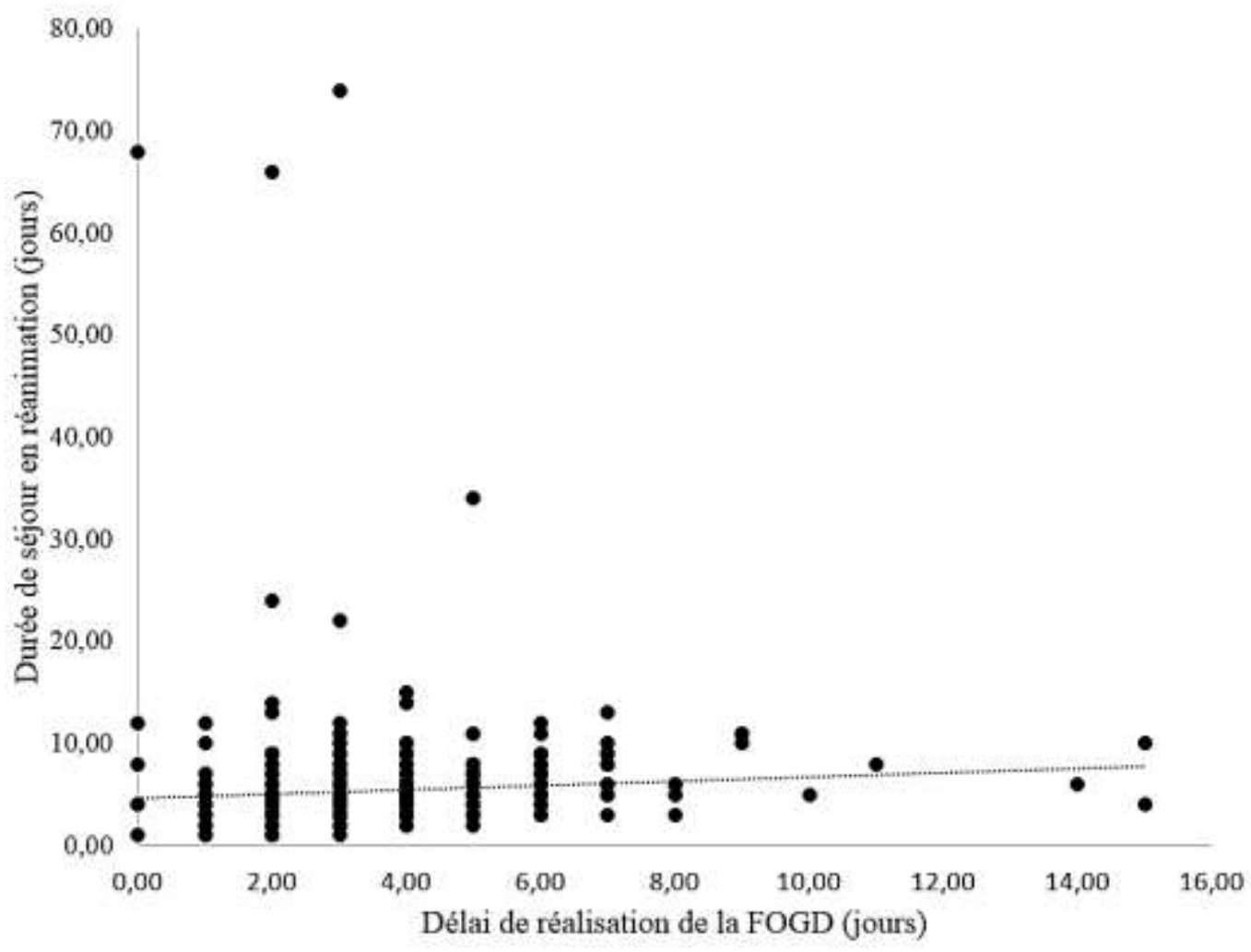

Figure 3:- Délai de réalisation de FOGD et durée de séjour. 


\section{Conflit D'interet:-}

Les auteurs ne déclarent aucun conflit d'intérêt.

\section{References:-}

1. Chen PH, et al. (2012): Delayed endoscopy increases re-bleeding and mortality in patients with hematemesis and active esophageal variceal bleeding: A cohort study. J. Hepatol., 57(6):1207-1213.

2. Cho SH, et al (2017): Outcomes and role of urgent endoscopy in high-risk patients with acute nonvariceal gastrointestinal bleeding. Clin Gastroenterol Hepatol., pii: S1542-3565(17)30736-X.

3. Cooper GS, et al. (1999): Early endoscopy in upper gastrointestinal hemorrhage: associations with recurrent bleeding, surgery, and length of hospital stay. Gastrointest. Endosc.,49(2):145-152.

4. Hsu YC, et al. (2009): Delayed endoscopy as a risk factor for in-hospital mortality in cirrhotic patients with acute variceal hemorrhage. J. Gastroenterol. Hepatol., 24(7) : 1294-1299.

5. Hsu YC, et al (2011): Application of endoscopy in improving survival of cirrhotic patients with acute variceal hemorrhage. Int J Hepatol., 2011:893973.

6. Hwang $\mathrm{JH}$, et al. and the Standards of Practice Committee of the American Society for Gastrointestinal Endoscopy. (2014): The role of endoscopy in the management of acute non-variceal upper GI bleeding. Gastrointest. Endosc., 75(6) : 1132-1138.

7. Kumar NL, et al. (2017) : Timing of upper endoscopy influences outcomes in patients with acute nonvariceal upper GI bleeding. Gastrointest. Endosc., 85(5):945-952.

8. Kumar NL, et al. (2016): Initial management and timing of endoscopy in nonvariceal upper GI bleeding. Gastrointest. Endosc., 84; (1) : 10-17.

9. Laursen SB, et al. (2017): Relationship between timing of endoscopy and mortality in patients with peptic ulcer bleeding: a nationwide cohort study. Gastrointest. Endosc., 85(5) : 936-944.

10. Lee JG, Turnipseed S, et al. (1999). Endoscopy-based triage significantly reduces hospitalization rates and costs of treating upper GI bleeding: a randomized controlled trial. Gastrointest. Endosc., 50(6):755-761;

11. Lim LG, et al. (2011): Urgent endoscopy is associated with lower mortality in high-risk but not low-risk nonvariceal upper gastrointestinal bleeding. Endoscopy 2011; 43(4): 300-306.

12. Myers RP, et al. (2009): The effect of weekend versus weekday admission on outcomes of esophageal variceal hemorrhage. Can. J. Gastroenterol., 23 (7): 495-501.

13. Sarin N, et al. (2009): Time to endoscopy and outcomes in upper gastrointestinal bleeding. Can. J. Gastroenterol., 23 (7) : 489-493.

14. Sonnenberg A. (2014): Timing of endoscopy in gastrointestinal bleeding. United European Gastroenterol. J., 2(1) 5-9.

15. Spiegel BM. (2009): Endoscopy for acute upper GI tract hemorrhage: sooner is better. Gastrointest. Endosc., 70 (2) : 236-239.

16. Targownik LE, et al. (2007) : The role of rapid endoscopy for high-risk patients with acute nonvariceal upper gastrointestinal bleeding. Can. J. Gastroenterol., 21(7) : 425-429.

17. Wong JCT, Lau JYW. (2017) : Is timing everything in the management of acute upper GI bleeding? Gastrointest. Endosc., 85(5):953-955.

18. Wysocki JD, et al. (2012): A nationwide analysis of risk factors for mortality and time to endoscopy in upper gastrointestinal haemorrhage. Aliment. Pharmacol. Ther., 36(1): 30-36. 